\title{
HACIA LA CONTEMPORANEIDAD: REFLEXIONES SOBRE LA EDUCACIÓN ARTÍSTICA EN LA DÉCADA DEL NOVENTA EN QUITO.
}

Pamela Cevallos

Manuel Kingman

\section{Resumen:}

Este artículo propone un acercamiento histórico sobre la educación universitaria de artes visuales en los años noventa, para analizar la incidencia de los debates y posiciones sobre el arte contemporáneo en el campo educativo. En los noventa surgió un discurso sobre lo contemporáneo en contraposición al quehacer moderno que articuló las prácticas artísticas y las posturas institucionales. Así, durante esta década, la educación universitaria en artes expresó las tensiones del campo: entre una formación basada en el oficio y la demanda de los jóvenes artistas por acceder a referentes y teorías contemporáneas.

Palabras claves: educación artística, universidad, posmodernidad, contemporaneidad, años noventa, Quito, FAUCE, CAV-PUCE

\begin{abstract}
:
This article introduces a historical approach on college education in visual arts during the 90's to explore the debates about contemporary art in the field of education. In the 90's the discussion about contemporary art against modern endeavors emerged, causing a discussion about contemporary art which developed certain artistic practices and institutional standpoints. In consequence during this decade arts' college education showed the strain between training based on craftsmanship, and the students' demand to access contemporary theories and references.
\end{abstract}

Keywords: art education, university, postmodernity, contemporaneity, nineties, Quito, FAUCE, CAV-PUCE

\author{
Autores: \\ Pamela Cevallos (Quito, 1984). Máster en Antropología Visual FLACSO y Licenciada en \\ Artes Plásticas UCE. Docente de la Carrera de Artes Visuales de la PUCE. \\ Manuel Kingman (Quito, 1976). Máster en Antropología Visual FLACSO y Licenciado en \\ Artes Plásticas PUCE. Docente de la Carrera de Artes Visuales de la PUCE.




\section{La contemporaneidad en el arte}

La inquietud sobre la educación artística parte de nuestra experiencia como estudiantes de arte entre 1997 y 2007 en las dos carreras y facultades revisadas en este texto y nuestra adscripción actual a la Carrera de Artes Visuales de la PUCE. En ese sentido, nos planteamos producir un ensayo que aporte tanto al reconocimiento de una parte de la historia de la educación artística del Ecuador y específicamente en Quito, como a su proyección, a través de las reformas que podamos generar en nuestros ámbitos de acción. El material de análisis para realizar este ensayo es la documentación de prensa de la década del noventa, y entrevistas a artistas que transitaron como estudiantes o profesores por las instituciones de educación artística ${ }^{1}$. Estamos conscientes que el alcance de este ensayo es limitado, sin embargo, consideramos importante aproximarnos al tema para aportar al debate en el actual momento de redefinición de las mallas curriculares de arte por los procesos de evaluación, categorización y acreditación de las carreras y facultades en las instituciones de educación superior ${ }^{2}$.

En este ensayo nos referiremos a la década del noventa como un momento central en la definición de lo contemporáneo en las artes, que planteó cuestionamientos en diversos ámbitos, incluyendo el educativo. Artistas y críticos

1 Este ensayo surge del proyecto de investigación "Modernidad y Contemporaneidad en el arte ecuatoriano de los años noventa", 2014-2015, auspiciado por la PUCE, que indaga en la emergencia de lo contemporáneo durante la década de 1990 desde la producción, circulación y consumo.

2 Conjuntamente con Camila Molestina formamos parte del Comité de Reforma Curricular de la Carrera de Artes Visuales de la PUCE. Los lineamientos de este proceso fueron generados por el Consejo de Evaluación, Acreditación y Aseguramiento de la Calidad de la Educación Superior (CEAACES). apelaban a la contemporaneidad en el arte desde distintos lugares y con repercusiones diferentes durante toda la década. Muchas de las críticas a la modernidad decantaron en renovaciones de salones y bienales ${ }^{3}$ y en la generación de instituciones e infraestructura para la investigación sobre el arte contemporáneo ${ }^{4}$. Si la presencia de lo contemporáneo en el arte de los noventa se traduce en nuevas sensibilidades y estrategias de producción que intentan marcar un corte con lo moderno, nos preguntamos ¿cómo respondió la academia a estos debates? En este sentido, analizaremos el campo del arte y específicamente la educación artística universitaria en Quito para comprender los distintos impulsos y contextos que evidencian cómo se comprendió el arte y la educación en la década del noventa y el grado de inmersión en el debate del arte contemporáneo 5 .

Para hablar de contemporaneidad en el arte es necesario situar al modernismo como

3 Nos referimos específicamente a la Bienal de Cuenca y al Salón Mariano Aguilera que en el 2001 y en el 2002 abrieron sus puertas a nuevos medios de producción artística.

4 El CEAC (Centro Ecuatoriano de Arte Contemporáneo), como institución independiente desde 1995, articuló un proyecto de investigación y de formación en debates artísticos contemporáneos. En el ámbito museal el MAAC (Museo de Arqueología y Arte Contemporáneo de Guayaquil) abre sus puertas en el 2002, en su apertura se perfila como un proyecto de investigación y coleccionismo en torno al arte contemporáneo del Ecuador.

5 María del Carmen Oleas (2015) propone pensar en la educación artística del Ecuador (específicamente la FAUCE y la CAV) a partir de dos cuerpos teóricos, el concepto de campo de Bourdieu y el de régimen de arte de Ranciere. Coincidimos con Oleas en que estos conceptos son útiles para pensar sobre el arte contemporáneo ecuatoriano, independientemente del mayor o menor grado de estructuración del campo del arte (Bourdieu, 2002) o la vigencia de tal o cual régimen del arte (Ranciere, 2009), hay que reconocer que estas nociones tienen que ser entendidas de manera específica de acuerdo con el contexto específico de Quito. 
un amplio paraguas que acoge a distintos movimientos ubicados temporalmente entre comienzos del siglo XIX y mediados del siglo XX. Se puede reconocer al modernismo en las vanguardias históricas; celebratorias de la razón instrumental, irónicas y críticas de las consecuencias del progreso. Según Harrison el modernismo se trata "de una forma de militancia y experimentación de carácter vanguardista. [...] se trata de una revolución cultural específica impulsada por el rápido progreso tecnológico y por la agitación política, que llevó consigo la búsqueda del cambio por el cambio y el ejercicio de una forma de militancia y experimentación de carácter vanguardista" (Harrison, 2000: 11). Aunque las experimentaciones y posturas políticas de los artistas modernos son diversas, hay ciertos preceptos que son recurrentes en la literatura sobre el arte moderno, estos elementos actúan como fundamentos de legitimación y consolidación del valor artístico. La noción de originalidad y cambio, la imaginación como garantía de la libertad humana, la conciencia autocrítica en las posibilidades propias de cada medio, la autonomía estética de la obra de arte y la genialidad del artista (Harrison, 2000). La narrativa más autárquica y grandilocuente del modernismo fue planteada por Clement Greenberg y sus discípulos, el autor pensaba al arte en la relación con una autocrítica y revisión de su propia disciplina medial y en un progreso continuo (Greenberg, 2002 [1965]).

Si la modernidad contemplaba un futuro utópico de mejoramiento de la sociedad, la posmodernidad coincide con el fin de los metarrelatos, la crisis de la subjetividad como baluarte de la sociedad y la muerte del autor. Las prácticas artísticas y la teoría del arte reaccionaron frente a nociones profundamente instituidas en el campo del arte moderno, como la noción de originalidad, la autonomía artística, el sitial elevado de un "Arte" con mayúsculas y el culto al genio creador (Krauss, 1996). A través de las posturas de críticos como Rosalind Krauss (1996) Douglas Crimp (2005), Craig Owens (1980), Fredric Jameson (2002[1984]) y Hal Foster (2001), entre otros, la teoría del arte posmoderno se articula como un sistemático desmantelamiento de la modernidad ${ }^{6}$.

Paralelamente a la discusión propiciada en los centros de producción artística, se generaron reflexiones transdisciplinarias que planteaban las relaciones entre cultura, geografías y poder desde América Latina. Autores como Gerardo Mosquera (1996), Mari Carmen Ramírez (1996) y Nelly Richard (1994), entre otros, desde sus contextos específicos propusieron repensar y superar el modelo hegemónico y eurocéntrico del "arte internacional". Sus análisis revisan las dicotomías centro-periferia, universal-local para cuestionar los cánones de internacionalización y la generación de otredad desde los flujos de ideas y prácticas de circulación y consumo.

La noción de contemporaneidad también se distancia de la modernidad, sin embargo, si la posmodernidad la cuestiona y deconstruye, la noción de arte contemporáneo articula otras preocupaciones relacionadas con la reflexión y problematización del presente (Terry Smith, 2013). Si el arte y el pensamiento postmodernos se centraban (sobre todo) en cuestionar los relatos modernos con la finalidad de desmantelarlos, el arte contemporáneo trata de

6 Por ejemplo, la teoría posmoderna estadounidense del arte también acompañó y cimentó la trayectoria de los artistas neo conceptuales y apropiacionistas de los ochenta, a la vez que impugnó a las prácticas artísticas pictóricas neo expresionistas criticando su adscripción al mercado del arte neo conservador de la era Reagan (Wallis, 2001). 
captar y problematizar un presente complejo y carente de metarrelatos, una contemporaneidad atravesada por lo local y lo global y en el que ya no son posibles las utopías sino los fragmentos de sentido. Andrea Giunta, en su reflexión centrada en Latinoamérica, sostiene la invalidez del esquema derivativo de centros y periferias para el estudio del arte contemporáneo. Por el contrario, se propone la noción de vanguardias simultáneas para analizar obras que se insertan en la lógica global del arte, pero que activan situaciones específicas (Giunta, 2014:5).

En los ańos noventa, en el contexto internacional y local se consolida una noción de lo contemporáneo en el arte. La teoría cultural y artística tuvo una repercusión innegable en las prácticas artísticas y en la academia, como veremos más adelante los contextos de formación artística de la ciudad respondieron de distintas maneras a estos debates sobre la posmodernidad y la contemporaneidad.

\section{Arte y paradigmas educativos}

El desarrollo de la educación artística universitaria en el Ecuador es parte de "procesos difusos, complejos e inestables del devenir de la nación" que no permiten una clara identificación "justamente porque las formas tradicionales de estudio, son superadas por los hechos y comportamientos que se desbordan y no pueden ser registrados en su totalidad" (Jaramillo, 2013: 58). Sin embargo, ¿bajo qué modelos o paradigmas se planteó la educación artística universitaria en el Ecuador? De acuerdo con el artista y profesor universitario Gonzalo Jaramillo (2013), quien ha investigado sobre el papel del taller de arte en la educación artística, no se puede hablar de un paradigma o sistema incorporado de manera totalmente coherente en las universidades ecuatorianas, no obstante, es necesario citar algunos de estos modelos y sus posibles comprensiones desde lo local.

Efland (2003) y Marín (1997) plantean que en la educación artística se han dado ciertos paradigmas. Efland sitúa a los modelos cronológicamente: el arte academicista (siglos XVII al XIX), Elementos del dibujo (principios del siglo XX) Expresión creativa del yo (de principios a mediados del siglo XX), Arte de la vida cotidiana (1930 a 1960) y el Arte como disciplina (1960 a 1990), mientras que, Marín establece cuatro modelos pero su comprensión de la enseñanza artística inicia en el taller de artista medieval, comprende el sistema de la Academia de Bellas Artes, a la Bauhaus y al sistema del genio o del desarrollo creativo personal (1997).

Desde los años ochenta, la teoría posmoderna permitió mirar críticamente el papel del artista moderno: la manera en que la expresión cultural se redujo a problemas de estilo, la noción de inspiración y el poder de creación divino y la imposibilidad de interpretar unívocamente las obras de arte. Es decir, "tanto la obra de arte como el artista pierden su posición de privilegio en tanto fuentes del saber" (Efland, 2003:124) ${ }^{7}$. En Estados Unidos, las discusiones sobre el posmodernismo y los parámetros de excelencia en la educación decantaron en un modelo basada en disciplinas (DBAE por sus siglas en inglés $)^{8}$ que llevaba a pensar el currículo de

7 En una línea similar a la de Efland, Parks traza las diferencias de la educación artística en la modernidad y en la posmodernidad, su texto, producido en 1989 evidencia el debate del momento, el autor muestra cómo en el modernismo se propende a un modelo de educación que privilegiaba la originalidad y el cambio, el rechazo del pasado, la individualidad y la autoexpresión (Parks, 1989: 11).

8 El modelo basado en disciplinas se generó en los años sesenta, durante los años ochenta hay una nueva 
educación artística de una manera organizada y estructurada que concebía al arte como un campo científico disciplinar (Efland, 2002: 371 - 373).

Mientras el debate sobre la excelencia y el arte como portador de conocimiento se estaba cimentando en las universidades americanas en las décadas del ochenta y noventa ¿qué se estaba debatiendo en las universidades locales?, ¿cómo se comprendía la educación artística? Antes de discutir sobre la presencia del debate posmoderno en la educación artística quiteña es necesario contextualizar los espacios de educación artística vigentes en esa época, con la advertencia también que estaban marcados por continuas crisis económicas y políticas neoliberales que también afectaron la calidad de la educación. Nos remitimos a dos casos que permiten poner en diálogo trayectorias institucionales distintas: en primer término, la Carrera de Artes Plásticas de la Facultad de Artes de la Universidad Central (FAUCE) fundada en 1968, y luego, la Carrera de Artes Plásticas -actualmente Carrera de Artes Visuales- de la Pontificia Universidad Católica (PUCE) que en 1997 abrió sus puertas?

\section{La FAUCE: entre la expresión y la emergencia de} lo contemporáneo

propuesta educativa basada en este concepto (Efland, 2002).

9 El alcance de la investigación no da la posibilidad de situar el papel que la Escuela de Artes de la Universidad de Cuenca tuvo en la formación de artistas; sin embargo, muchos artistas contemporáneos se formaron en la misma. Esta escuela se constituye en el año 1988 a partir de la transformación de la Escuela de Bellas Artes en Escuela Superior de Artes adscrita a la Facultad de Arquitectura. Esta escuela se consolida en el año 2000 como Facultad de Artes. La Carrera de Artes de la Universidad San Francisco comienza en 1988, pasa por algunas Facultades y desde el 2004 forma parte del COCOA (Colegio de Comunicación y Artes Contemporáneas).
La Carrera de Artes Plásticas de la FAUCE, desde su fundación, se constituyó en la institución educativa más importante del país y la única en ofertar títulos de tercer nivel en el ámbito artístico, esta institución convocaba a jóvenes de distintas provincias que llegaban a Quito a realizar sus estudios. Sin embargo, desde sus inicios, experimentó una fuerte inestabilidad política y académica. Su estructura previa como Escuela de Bellas Artes (desde 1904) de alguna manera se mantuvo en un pensum articulado en las técnicas tradicionales: pintura, escultura y grabado ${ }^{10}$. En su investigación sobre la historia de la Facultad de Artes, la artista y docente Jenny Jaramillo señala además que en su caótica fundación en 1968, se mantuvieron profesores de la Escuela de Bellas Artes que basaban el objetivo de enseñanza en el dominio de las técnicas y métodos de la historia clásica y moderna de la pintura y escultura (Jaramillo, 2014:55).

Durante las décadas de 1970 y 1980, la FAUCE fue modificándose hacia un tipo de formación especializada que implicaba una valoración sobre las libertades de los artistas y la exploración en las posibilidades expresivas de los medios (Jaramillo, 2014: 90). Pero es en la década de 1990 donde se ubica un quiebre con el paradigma modernista que había marcado la educación en la Facultad de Artes. Para Jaramillo, la aparición de manifestaciones de desborde y que, paradójicamente, conlleva a la hibridación y expansión de las prácticas, está relacionada con la repercusión del abstraccionismo abstracto y la hegemonía cultural norteamericana expresada en el proceso de globalización comunicacional (Jaramillo, 2014:92). En este sentido, fue clave

10 A pesar de contar con una estructura basada en técnicas tradicionales (pintura, escultura y grabado), su objetivo no fue consolidar un paradigma academicista regido por las normas “clásicas” del arte. 
la incorporación de profesores como Mauricio Bueno y, posteriormente, de Pablo Barriga y Juan Ormaza que refrescaron el tradicionalismo y las discusiones que marcaban la enseñanza en la Facultad. Estos artistas formados en Estados Unidos, Inglaterra y México, introdujeron planteamientos desde el conceptualismo y los nuevos medios ${ }^{11}$.

No obstante, en los noventa este espacio académico todavía se autodefinía por su oferta de "oficio" a los jóvenes artistas y su éxito se medía por la cantidad de premios de estudiantes y profesores obtenidos en salones y concursos a nivel nacional (El Comercio, 21 de febrero de 1993). Por ejemplo, en una entrevista realizada a mediados de los noventa a varios profesores, se debatía sobre la importancia de formación del oficio y sobre si la creatividad era importante o no en la formación universitaria, para algunos profesores la creatividad era relevante pero en los últimos niveles, la mención del valor de las ideas y los conceptos en la formación artística estaban ausentes de ese debate específico (El Comercio, 23 de febrero de 1995). Además, algunos profesores se referían a ciertos lenguajes como la instalación, el performance y el video como modas pasajeras a pesar que estaban validadas por el campo artístico desde los sesenta. (El Comercio, 4 de enero de 1995).

Si la estructura académica de la FAUCE en ciertos sentidos era tradicionalista, algunos artistas que se formaban en ese espacio, estaban proponiendo prácticas distintas que se ampliaban hacia nuevas posibilidades de producción artística. Así, podemos mencionar

11 Mauricio Bueno estudió en Massachusetts Institute of Technology en Estados Unidos, Pablo Barriga en Saint Martin School of Artes en Inglaterra y Juan Ormarza en la Escuela Nacional de Arte "La Esmeralda" en México y en Alfred University en Estados Unidos. por ejemplo algunas propuestas que expandían las propias posibilidades del medio pictórico, cuestionando y ampliando sus límites (Pablo Barriga, "Todo hace pensar que son ellos",1988) y desmaterializando sus soportes (Pilar Flores, "Montaña Mar", 1994), y rompían con la tradición pictórica preponderante a través del performance, (Jenny Jaramillo, "Piel, Pared, Galleta”, 1995) Así, en el transcurso de la década la noción de lo contemporáneo se reafirma en oposición a lo moderno y al aura del objeto. Artistas que se formaron en la FAUCE como Danilo Zamora, Patricio Ponce y Jenny Jaramillo, que trabajaban desde la pintura expandida y el performance, eran críticos con una formación que privilegiaba el desarrollo técnico por sobre el posicionamiento reflexivo (Jaramillo y Ponce, entrevista, 2014). El cuestionamiento hacia la formación recibida, motivaba un choque con el arte moderno, en tanto que era el arte legitimado e institucionalizado y que tenía salida en el mercado (Zamora, s/f).

En el proceso de formación, además de los referentes proporcionados por los profesores, existía una necesidad de autoformación que revela la importancia que los artistas emergentes dieron al aspecto teórico. Esto se evidencia en los seminarios organizados por el CEAC, colectivo independiente que acogió el término "contemporáneo" para marcar un deber ser de las prácticas artísticas de ese momento. El CEAC se vinculó desde 1996 a la crítica e historiadora del arte cubana Lupe Álvarez quien aportó con seminarios, talleres y cursos de especialización que abordaban el trance a lo contemporáneo ${ }^{12}$.

12 Había una necesidad de autoeducación por parte de los artistas, que aprovechaba la circulación de revistas como "Art in America" y de los viajes de los artistas para actualizarse en referentes contemporáneos que luego eran distribuidos en fotocopias. Entre los seminarios y talleres impartidos por Álvarez estan: "Arte en 
Álvarez (2000) tachó de patético el nivel de exigencia teórico en los pensums. Decía: "En las escuelas abunda la idea de que la ignorancia es síntoma de pureza, (...)" (Álvarez, 2000). Es interesante observar que entre los textos distribuidos a los estudiantes durante estos seminarios se encuentran predominantemente autores relacionados con enfoques críticos y postmodernos.

Por ejemplo, en el Seminario "Problemas Teóricos sobre la Enseñanza del Arte" (realizado entre marzo y abril de 1997) se distribuían textos de autores como Pierre Bourdieu, Fredric Jameson, Stefan Morawski, Michal Glowinski, Allan O'Connor, Gerardo Mosquera, Gianni Vattimo, Jean Claude, Passeron, Hal Foster, Louis Marin, Felix Guattari, Ricardo Subercaseaux, Arthur Danto, Nelly Richard, Andreas Huyssen entre otros (CEAC, s/f). En el año 2000, un taller importante generado desde la propia Facultad de Artes de la Universidad Central, en colaboración con Artes No Decorativas S.A, fue el seminario "Sacando Punta a la Materia Arte" este taller coordinado por el artista cubano Saidel Brito, dio herramientas conceptuales a los estudiantes de último nivel de la facultad, los resultados de este seminario se expusieron en la muestra "Mil cuatrocientos ochenta y seis kg", en esta exposición se evidenció un giro -tanto en el aspecto medial como conceptual- en el tipo de propuestas presentadas (Catálogo "Mil cuatrocientos ochenta y seis Kg", 2001).

trance o ¿Qvo vadis ars?” (1996), "La fotografía en la cultura contemporánea"(1996), "Problemas teóricos de la enseñanza del arte" (1997), "Autoconciencia creativa y perspectiva crítica" (1997), El espacio y la idea. Curando los nuevos significados del arte (1998) ¿Cómo hallarle sentido a la crítica de arte sin perecer en el intento? (1998) El Cine latinoamericano ante el nuevo milenio (1998). El curador inglés Kevin Powers, especializado en arte latinoamericano también dictó conferencias en el país (El Comercio, 8 agosto de 1999).
En la FAUCE, el paradigma educativo preponderante consideraba como máximo valor la originalidad exclusiva de la expresión individual (Efland, 2003:120). Paradójicamente este paradigma potenció en los años noventa prácticas que expandieron una noción de arte modernista hacia lo contemporáneo. A partir de los aportes de profesores concretos de la FAUCE y de la circulación de información sobre arte contemporáneo se dinamizaron los debates y las prácticas por fuera del espacio de formación, es decir, si bien no una implicó transformación sustancial en el pensum de la carrera, muchos artistas que pasaron por las aulas de la FAUCE, contribuyeron con propuestas que ampliaron las posibilidades de creación artística, sus procesos de producción artística también aportaron a la inclusión de lenguajes y debates contemporáneos en otras academias e instituciones culturales.

\section{Carrera de Artes Visuales de la PUCE: Debates posmodernos y formación artística}

\section{La Carrera de Artes Plásticas de la PUCE se fundó} en septiembre de 1997, como parte la Facultad de Arquitectura y Diseño. Esta Facultad, creada en 1994, proponía un modelo educativo basado en "aprender haciendo" y una formación integral y de complementariedad entre las carreras, por ello desde el inicio se proyectó la creación de la Carrera de Artes Plásticas como una parte importante de la FAD (Vásconez, 2014) ${ }^{13}$. En la Propuesta de la Carrera de Artes Plásticas (mayo 1997) se expone sobre todo el aspecto pedagógico (aprender - haciendo) y la relación

13 La importancia otorgada al ámbito artístico desde la FAD, se evidencia en una serie de publicaciones sobre fotografía artística contemporánea que incluyó la obra de Lucía Chiriboga, Judy Bustamante, Mimmo Privitera, Pepe Avilés y Diego Cifuentes, estos tres últimos eran profesores de la Facultad. Buscar documento. 
del artista con la sociedad, este documento fundacional da unas pautas a seguir y ya plantea la estructuración del pensum a través de talleres práctico - reflexivos (Propuesta Carrera de Artes Plásticas, 1997).

A partir de los testimonios y memorias de los graduados y la lectura de otros documentos posteriores se puede afirmar que en un principio la visión educativa de la carrera se fundamentó en el incentivo de la creatividad y la expresividad, un modelo cercano al categorizado como "expresión creativa del yo" (Efland, 2003). Esta visión también se evidencia en las materias que se dictaban, pues además de las disciplinarias (Dibujo, Pintura, Grabado, Taller de Arte, Historia y Teoría del Arte) existían materias de expresión corporal, sensibilización a la música y se realizaban salidas de campo con énfasis en el estímulo de la creatividad (Gonzalo Vargas, entrevista, 2015). En un fragmento de la propuesta de 1998 se encuentra la siguiente frase, la cual dice mucho del tipo de artista que se quería formar:

\footnotetext{
"Nuestra escuela de Artes plantea como eje principal el contacto del ser humano con la naturaleza y con su realidad. Se busca de este modo llegar a la sensibilización y al autoconocimiento de los alumnos a través de las fuerzas generadoras de vida. Ya que la conciencia humana, la capacidad de reflexión y creación se desarrollan paulatinamente mediante la conquista de esta realidad." (Propuesta Carrera de Artes Plásticas, 1998)
}

Luego del primer semestre de funcionamiento, la llegada de nuevos profesores contribuyó a la definición de una metodología pedagógica basada en el Taller de Arte. A diferencia de la FAUCE que estaba estructurada a partir del perfeccionamiento técnico y la expresión a través de medios tradicionales; el planteamiento de la Carrera de Artes de la PUCE proponía al Taller de Arte como un espacio para trabajar a través de problemáticas específicas que podían ser abordadas desde distintos medios. En el pensum de 1999 se define la materia Taller de Arte (6 créditos) como un eje central teórico práctico. Metodológicamente el Taller de Arte estaba basado en ejercicios, el profesor proponía un problema que tenía que ser resuelto por los estudiantes con el medio más idóneo para cada caso (Descripción sintética de cursos 99: 1999).

Según Gonzalo Jaramillo (entrevista, 2014), quien fue profesor y Director de la Carrera de Artes Plásticas, artistas y teóricos activos en el campo confluyeron en este espacio y aportaron con una visión contemporánea del arte $^{14}$. Aunque tal vez sin una conciencia en ese hecho, se estaba pasando de un modelo basado en el estímulo de la "expresión", a un modelo basado en disciplinas (Efland, 2002); sin embargo, ciertos aspectos del anterior modelo siguieron presentes en la formación artística. Uno de los momentos más interesante de la Carrera de Artes de la PUCE se ubica entre 1999 y principios del 2001, bajo la dirección de Enrique Vásconez hay un ambiente muy productivo y una efervescencia en las aulas, además, se perfila la consolidación de un proyecto de carrera que cuenta en su planta con artistas comprometidos con el arte contemporáneo. Ese proyecto se

14 Entre los profesores vinculados a la PUCE en la primera etapa constan Gonzalo Jaramillo, Pilar Flores, Enrique Vásconez, Pablo Barriga, Marcelo Aguirre, Ana Rodríguez, María del Carmen Carrión, José Avilés, Alexis Moreano, Rosa Jijón, entre otros. Como un ejemplo del tipo de enfoque, podemos nombrar que Historia del Arte era dictada por Pablo Barriga, como uno de los artistas precursores del arte contemporáneo en el Ecuador; este artista proponía una lectura crítica y contextualizada del arte; Alexis Moreano (miembro activo del CEAC) enseñaba Teoría del Arte e introducía en las nociones de la posmodernidad en el arte. 
frustra en mayo del 2001, la FAD sufre una crisis, el Rector padre José Ribadeneira S.J altera el modelo administrativo y educativo de la Facultad y en ese proceso se acepta la renuncia de Enrique Vásconez y otros profesores de Artes (Vásconez, 2014).

Durante muchos años la Carrera de Artes Plásticas queda a la deriva, hay un período de inestabilidad y fragmentación, bajo la administración del cubano Jorge Alberto Gómez, quien trata de imponer un método educativo de corte tradicional (entrevista, Gonzalo Jaramillo, 2014). Recién en el 2004, bajo la Dirección de Alfredo Breilh, la Carrera de Artes Plásticas de la PUCE, genera un rediseño curricular a partir de un diagnóstico interno que observaba su funcionamiento y proyección en los últimos cinco años. Uno de los principales problemas que enfrentaba era la invisibilidad de la carrera y el hecho que su denominación no guardaba relación con las competencias esperadas de los graduados. Así, la carrera cambió de nombre para dar mayor énfasis en las Artes Visuales Contemporáneas. $\mathrm{Al}$ respecto de los valores diferenciales de este nuevo planteamiento se propuso ir "más allá del perfil de Artes Plásticas tradicionalmente conocido y desarrollado" y ampliar el campo de acción "a las nuevas formas de expresión del arte visual contemporáneo, incluyendo los diversos soportes tecnológicos" (Proyecto de Reforma Curricular, 2004). En el documento se menciona el vídeo artístico, la fotografía digitalizada, la multimedia, el performance, entre otros. También se ofertan nuevas perspectivas laborales para estudiantes de arte como la docencia, la crítica de arte, la curaduría y la gestión cultural.

Coincidimos con Gonzalo Jaramillo (2013) sobre la imposibilidad de hablar de modelos totalmente estructurados para el caso de la Carrera de Artes Visuales. Se trataría más bien de momentos en los que primaron distintos tipos de educación sin excluir la convivencia de materias con diversos enfoques. El giro de un modelo expresivo de la educación hacia una educación empapada de las discusiones posmodernas y nociones del arte contemporáneo, ya se fue incorporando en los contenidos de las materias desde el segundo semestre de funcionamiento de la Carrera de Artes Visuales. Aunque en el 2004 se creó una malla curricular que se proyectaba desde la noción de arte contemporáneo, esta aproximación ya se había implementado desde los primeros años de funcionamiento a partir de los aportes e inquietudes de los profesores.

La crisis de la carrera de Artes Visuales en mayo del 2001 perjudicó la calidad educativa y el impulso como un proyecto de formación; sin embargo, se puede afirmar que es un espacio que oxigena al medio artístico quiteño y amplía las posibilidades de producción artística. En agosto del 2001, meses después de la crisis institucional de la Facultad de Arquitectura, Diseño y Artes, la Carrera propone la exposición "Amnesia", la cual se produce a partir de un concepto y articula a otras universidades como la FAUCE y Carrera de Artes de la San Francisco (El Comercio, 18 de agosto de 2001). Este caso evidencia el impulso por construir procesos de intercambio artístico entre las instituciones de formación artística.

\section{Reflexiones finales}

Las Carreras de Artes Plásticas de la FAUCE y la PUCE son casos emblemáticos para pensar el campo de la educación artística universitaria en Ecuador que, aunque han sido escasamente estudiados, contribuyen a la comprensión del campo artístico y las dinámicas del arte 
contemporáneo. En conclusión, se puede expresar que en la década del noventa se expresan permanentemente tensiones entre concepciones modernas y contemporáneas sobre el arte, que repercuten en el ámbito de la formación artística, dentro y fuera de las estructuras institucionales.

En este ensayo, hemos introducido ciertas reflexiones iniciales sobre las relaciones entre los paradigmas educativos que caracterizaron a las carreras de arte y la emergencia del arte contemporáneo en la década del noventa. Sin traspasar nociones occidentales sobre paradigmas educativos al escenario ecuatoriano, vemos que la expresividad en el arte es uno de los rasgos más claros de la FAUCE. Así, el paradigma de la "expresión creativa del yo" (Efland, 2003) o "el sistema del genio" (Marín, 1997), se acercaría a un tipo de concepción institucional que fue cuestionada permanente desde las prácticas artísticas emergentes que apelaban a lo contemporáneo para expandir el propio campo artístico. Consideramos que la fundación de la Carrera de Artes Plásticas de la PUCE es parte de este impulso de expansión del campo y de las discusiones y acciones por renovar el campo artístico. En ambos casos, se puede pensar en la filtración de nociones críticas a la modernidad a través de profesores y estudiantes cercanos a las teorías de la posmodernidad y comprometidos con la contemporaneidad artística.

Consideramos que, una pregunta de investigación para la profundización de este tema debería enfocarse en cómo los actores y sus prácticas artísticas y discursivas, paulatinamente se insertaron y reconfiguraron el ámbito educativo. En la actualidad tanto la Carrera de Artes Visuales de la PUCE como la Carrera de Artes Plásticas de la UCE tienen el reto de rediseñar sus mallas curriculares, es necesario que revisen críticamente sus propias trayectorias cuestionando el papel del arte en la sociedad actual. Aunque los dos espacios han tenido trayectorias separadas, muchos de los artistas formados en la Universidad Central han aportado a la Carrera de Artes Visuales de la PUCE. En la actualidad, muchos de los artistas, educadores y gestores que forman parte del campo del arte ecuatoriano se han graduado en estas dos instituciones. En este sentido, es importante ahondar en una mirada histórica a los procesos educativos que contribuyan a la consolidación de espacios de diálogo y proyectos en conjunto.

\section{Bibliografía}

Álvarez, L., (2001) "Antigüedades recientes en el arte ecuatoriano", Políticas de la diferencia, Arte iberoamericano de fin de siglo, Valencia: Generalitat Valenciana.

Bourdieu, P., (2002) Campo de poder, campo intelectual. Buenos Aires: Montessor.

Efland, A., (2003). La educación en el arte posmoderno. Barcelona: Paidós.

Efland, A., (2002). Una historia de la educación del arte: Tendencias intelectuales y sociales en la enseñanza de las artes visuales. Barcelona: Paidós.

Crimp, D., (2005) Posiciones críticas: ensayos sobre las políticas de arte y la identidad Madrid: Akal.

Foster H., (2001). Asunto: Post, En Wallis, Brian Qué falla en esta imagen. Brian Wallis (ed) En Arte después de la modernidad: Nuevos planteamientos en torno a la representación. Madrid: Akal Ediciones, pp: 189-205.

Greenberg C., (2002 [1939]) "Modernist 
Painting” En Art in Theory, 1900- 2000: an anthology of changing ideas. Charles Harrison y Paul Wood, (Comps. USA: Blackwell

Publishing.pp: 773-779.

Harrison, C., (2000) Modernismo: Movimientos en el Arte Moderno. Hong Kong: Ediciones Encuentro.

Jameson, F., (2002 [1984]) The deconstruction of expression. En En Art in Theory, 19002000: an anthology of changing ideas. Charles Harrison y Paul Wood, (Comps. USA: Blackwell Publishing.pp:1046-1051.

Jaramillo, G., (2013) El taller de artes visuales, lugar de la investigación y creación, Quito.

Jaramillo, J., (2014) Medio artístico, trayectoria profesional y performance, Tesis para obtener el título de Maestría en Antropología Visual y Documental Antropológico, FLACSOEcuador, Recuperado de http://hdl.handle. net/10469/6790

Krauss, R., (1996) La originalidad de la vanguardia y otros mitos modernos.Madrid: Alianza.

Marín Viadel, R., (1997). "Enseñanza y aprendizaje en Bellas Artes: una revisión de los cuatro modelos históricos desde una perspectiva contemporánea". Arte, Individuo Y Sociedad, (9), 55.

Mosquera, G., (1996). Beyond the Fantastic: Contemporary Art Criticism from Latin America. Edited by Gerardo Mosquera. London: Institute of international Visual Arts.

Oleas Rueda, M., (2015) Cuerpos teóricos para revisar el arte contemporáneo en Quito (1967-

2007). El caso de la educación universitaria, Estudios Avanzados, $\mathrm{N}^{\circ}$ 23, julio 2015, ISSN0718-5014, IDEA-USACH, Santiago de Chile, pp. 80-94

Owens, C., (1980) The Allegorical Impulse: Toward a Theory of Postmodernism, October, Vol. 12 (Spring, 1980), pp. 67-86. Recuperado de http://www.jstor.org/stable/778575
Parks, M. E., (1989) Art Education in a PostModern Age en Art Education, Vol. 42, No. 2 (Mar., 1989), pp. 10-13.

Ramírez, M., (1996) Beyond "The Fantastic": Framing Identity in U. S. Exhibitions of Latin American Art, Art Journal, Vol. 51, No. 4, Latin American Art (Winter, 1992), en Beyond the Fantastic: Contemporary Art Criticism from Latin America, Mosquera, G. ed., Cambridge, MA: The MIT Press, pp. 60-68.

Rancière, J., (2009) El reparto de lo sensible. Santiago de Chile: LOM editores.

Richard, N., (1994) La puesta en escena internacional del arte latinoamericano: Montaje, representación, en Visiones comparativas: XVII Coloquio Internacional de Historia del Arte, 1011-1016. Mexico City: Universidad Nacional Autónoma de México, Instituto de Investigaciones Estéticas.

Smith, T., (2013). ¿Qué es el arte contemporáneo?. Buenos Aires: Siglo XXI Editores.

Taylor, B., (2000) Arte hoy,Madrid : Akal.

Vásconez, M., (2014) Ecuador 66: Cerrada por remodelación: Crisis en la Facultad de Arquitectura y Diseño de la Universidad Católica - 2001. Recuperado de http://mariovasconez. blogspot.com/2014/09/ecuador-66-cerradapor-remodelacion.html

Zamora, D., (s/f) El arte de conceptos en Ecuador. Los nuevos lenguajes en el arte ecuatoriano. En Revista Cuadrilatero: Recuperado de dhttp://www.damiantoro.com/ cuadrilatero/index.php?idSeccion $=135$

\section{Documentos:}

FADA-PUCE (2004) Proyecto de reforma curricular para la Carrera de Artes Plásticas, 
Quito.

99.

(1999) Descripción sintética de cursos

(1998) Propuesta Carrera de Artes Plásticas.

(1997) Propuesta Carrera de Artes

Plásticas.

CEAC (s/f) Problemas Teóricos de la Enseñanza del Arte, Autoconciencia Creativa y Perspectiva Crítica. Recopilación de Textos de Apoyo para cursos del CEAC.

Catálogo de la exposición "Mil cuatrocientos ochenta y seis $\mathrm{Kg}$ ", del 20 de febrero al 16 de marzo de 2001, Quito: Editorial Casa de la Cultura Ecuatoriana.

"Formar gente con oficio" (1993, febrero 25) El Comercio, p. B5.

"Exposición de las mil obras" (1995, febrero 23) El Comercio, p. C6.

"Hacia un arte mal elaborado" (1993, enero 4) El Comercio, p. 6B.

"La amnesia a través de 14 miradas" (2001, agosto, 18), El Comercio 7B

\section{Entrevistas:}

Gonzalo Jaramillo

Jenny Jaramillo

Patricio Ponce

Gonzalo Vargas 